\title{
Koebnerization and Eruptive Xanthomas
}

\author{
Michael A. Santos, $M D^{7}$ and Galen Foulke, $M D^{2}$

'Department of Medicine, WellSpan Good Samaritan Hospital, Lebanon, PA, USA; '²epartment of Dermatology, Penn State Hershey Medical Center, Hershey, PA, USA.

KEYWORDS: clinical image; eruptive xanthomas; koebnerization; Koebner phenomenon.

J Gen Intern Med 34(9):1947-8

DOI: $10.1007 / \mathrm{s} 11606-019-05178-1$

(C) Society of General Internal Medicine 2019

A 20-year-old man with Asperger syndrome, uncontrolled type 2 diabetes mellitus, and familial hypertriglyceridemia presented with pruritic papules that increased in number during periods of medication nonadherence. Skin exam showed diffuse, firm, yellowish papules with an erythematous halo on his back, chest, and extremities. Koebnerization was noted on his left arm and hand from scratching (Figs. 1 and 2). Over the previous 7 years, serum triglycerides ranged from 365 to $7,000 \mathrm{mg} / \mathrm{dl}$ (normal, 0$150 \mathrm{mg} / \mathrm{dl}$ ). Hemoglobin A1c was 14.9\%.

Eruptive xanthomas are 1-4-mm yellow-red papules that form on the extensor surfaces of the arms, legs, buttocks, and hands. ${ }^{1}$ Eruptions can be tender and pruritic, often associated with uncontrolled diabetes mellitus and severe hypertriglyceridemia. Lesions regress with treatment of the underlying disease and diet modification. ${ }^{2}$

Originally described in psoriasis, Koebner phenomenon (KP) manifests as new lesions of an existing skin condition following trauma to uninvolved skin. The eruption appears in the geometric areas affected by trauma. Koebnerization may occur as quickly as 3 days after the initial trauma, and up to 2 years later. The mechanism underlying KP is not well understood. KP is seen most often in psoriasis, lichen planus, vitiligo, flat warts, and keratosis follicularis (Darier's disease), and less commonly with eruptive xanthomas. ${ }^{3,4}$ This patient's lesions regressed with intensive triglyceride and glycemic control.

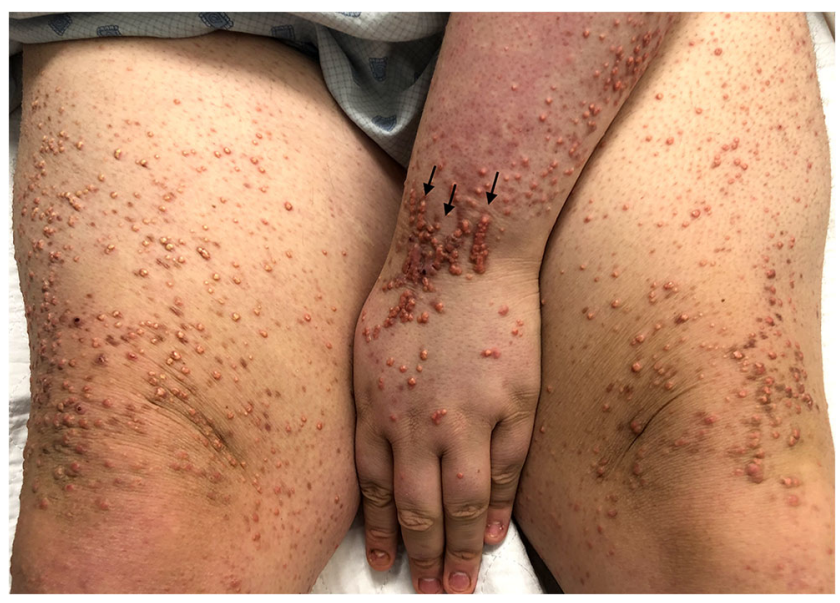

Figure 1 Eruptive xanthomas, appearing as yellow-red papules and nodules over the forearm, dorsal hand, extensor thighs, and knees. Arrows indicate koebnerization, with linear eruption of papules induced by scratching.

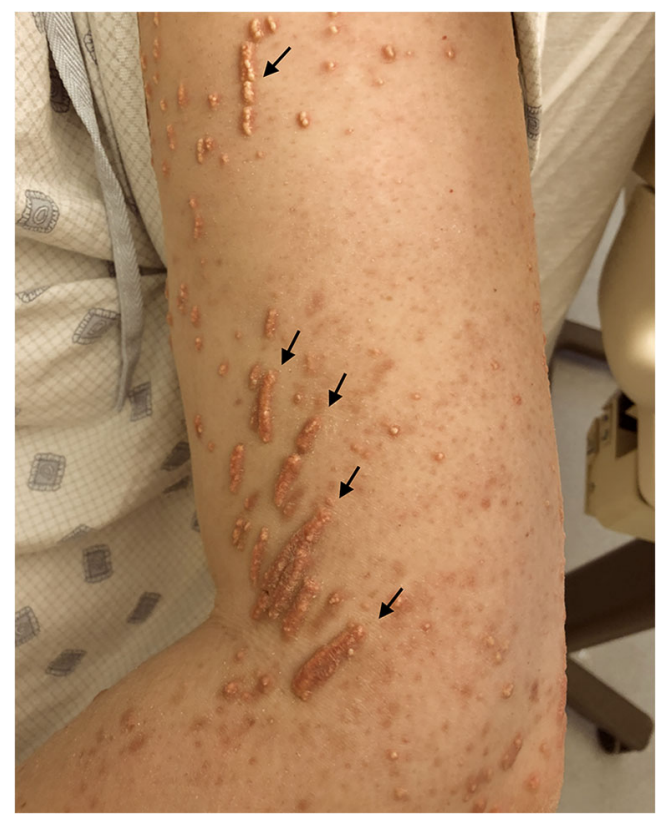

Figure 2 Koebnerized eruptive xanthomas on the lateral left arm. Linear yellow-red plaques appeared in areas of scratching. 
Corresponding Author: Michael A. Santos, MD; Department of Medicine WellSpan Good Samaritan Hospital, Lebanon, PA, USA (e-mail: msantos6@wellspan.org).

\section{Compliance with Ethical Standards:}

Conflict of Interest: The authors declare that they do not have a conflict of interest.

\section{REFERENCES}

1. Abdelghany M, Massoud S. Eruptive xanthoma. Cleve Clin J Med. 2015;82:209-10.

2. Parker F. Xanthomas and hyperlipidemias. J Am Acad Dermatol. 1985; 13:1-30

3. Naik NS. Eruptive xanthomas. Dermatol Online J. 2001;7:11.

4. Miller DM, Brodell RT. Eruptive xanthomatosis with linear koebnerization. J Am Acad Dermatol. 1995;33:834-5.

Publisher's Note Springer Nature remains neutral with regard to jurisdictional claims in published maps and institutional affiliations. 